\title{
A Biologically-Inspired Model for Recognition of Overlapped Patterns
}

\author{
Mohammad Saifullah \\ Department of Computer and Information Science \\ Linkoping University, Sweden \\ Mohammad.saifullah@liu.se
}

\begin{abstract}
In this paper a biologically-inspired model for recognition of overlapped patterns is proposed. Information processing in the two visual information processing pathways, i.e., the dorsal and the ventral pathway, is modeled as a solution to the problem. We hypothesize that dorsal pathway, in addition to encoding the spatial information, learns the shape representation of the patterns and, later uses this knowledge as a top-down guidance signal to segment the bottom-up, image-based saliency map. This process of segmentation in the dorsal pathway is implemented as an interactive process, where interaction between bottom-up image information and top-down shape cues lead to incremental development of a segmented saliency map for one of the overlapped object at a time. This segmented map encodes spatial as well as shape information of the respective pattern in the input. The interaction of the dorsal channel with the ventral channel leads to modulation and selective processing of the respective pattern in the ventral pathway for final recognition. Simulation results support the presented hypothesis as well as effectiveness of the model in providing a solution to the recognition of overlapped patterns. The behavior of the model is in accordance to the known human behavior on the occluded patterns.
\end{abstract}

Keywords: Vision, Attention, Neural network model, Overlapped patterns, Biologically-inspired, Saliency map, Interactive process.

\section{Introduction}

Recognition of overlapped pattern is a complex computational problem. This problem involves two fundamental issues in the field of machine vision, i.e., pattern segmentation and pattern recognition. The main difficulty in the recognition of overlapped patterns is accurate segmentation of individual patterns. If the segmentation step can be performed satisfactorily, the recognition of patterns becomes relatively easier task.

Traditional visual information processing theories [1][2][3], consider the segmentation and recognition as two independent process and, suggest that segmentation proceeds and facilitates recognition. In the same way, most of the 


\section{田田|F|F|B}

Fig. 1. Overlapped patterns

computational vision models [2][4][5] tackle the problem of overlapped patterns with a bottom-up 'Recognition followed by segmentation' approach. In this approach segmentation is performed by using image-based information only. As these approaches rely on only image-based cues for segmentation process, therefore, these bottom-up approaches cannot perform segmentation satisfactorily in the absence of such cues. There are evidences from the human vision studies [6][7] that high-level, class specific cues play an important role in image segmentation and thus endorse an interactive processing approach [8][9].

Consider the fig 1 , the pattern shown in the first image (from left) does not make much sense and cannot be named or recognized as a specific pattern. Now, look at the next three images in the sequence. These images are in fact the same pattern as in the first image, but due to bottom-up intensities based cues can be interpreted in the three images as overlap of the patterns 'TH', 'FFI' and 'FH' respectively. This time recognition becomes possible due to the presence of bottom-up cues that facilitate segmentation of overlapped patterns into individual patterns. Similarly, the last image also has no significant bottom-up cues that help segmentation and therefore, it is difficult for an artificial system, based on bottom-up approach, to correctly recognize the pattern in the image. This image can be interpreted as pattern ' $B$ ' or ' 13 ' depending on the high level knowledge, i.e., whether the pattern is English alphabet or overlapped digits. We may also notice that while interpreting the image as a overlapped of digits, we predict the digit and then move our focus along the patterns. Same is true in case of interpreting the pattern as a character pattern. From these images, we get a little insight that: i) Bottom-up cue play an important role in interpretation of the overlapped patterns, ii) In the absence of bottom-up as well as top-down cues, it is difficult to interpret overlapped patterns, iii) In the absence of bottom-up cues, the bottom-up, image-based information is interpreted with the help of top-down cues, in an interactive manner. In this paper, we deal with images of overlapped patterns which have little bottom-up cues. We use an interactive processing approach in which high level object knowledge guide the low level processing for segmentation and recognition of these patterns.

Now consider the case of humans. Humans have very good object recognition ability under large variations in conditions. In the event of many objects in the visual field, the visual systems use a sequential 'divide and conquer strategy' to recognize the objects one by one. In this strategy the main role is performed by the phenomenon of visual attention that helps select and process one object at a time. The phenomenon of visual attention can be best understood in terms of two stream hypothesis [10]. These streams are the ventral stream or 'What' pathway and the dorsal stream or 'Where' pathway. The ventral stream is responsible for recognizing and identifying objects while the dorsal deals with the spatial information associated with objects. 
The interaction between these two pathway lead to focus of attention on one of the many objects present in the scene. In this work we hypothesize that the dorsal channel learns the shape knowledge of the patterns and uses this information to perform segmentation of the overlapped patterns as an interactive process. Recent studies in neuroscience [11] [12] indicates that neurons in lateral intraparietal cortex (LIP) part of the dorsal pathway show some selectivity for shapes of the patterns, and hence suggest the possibility of encoding shape information along the dorsal pathway.

Most of the neural network models have used the bottom-up approaches for image segmentation. Mozer and colleagues [13][14] proposed a neural network model to segment overlapped objects. The network learns the underlying grouping principal to segment the objects. This model is a basically a bottom-up approach as it does not utilize any object knowledge to guide segmentation. Grossberg [15] presented a biologically-inspired model of the visual cortex that approaches the figure-ground separation as an interactive process. Another biologically-based neural network model is MAGIC [16], developed by Behrmann and colleagues, which uses bidirectional Connections to model grouping of features into objects within the ventral pathway. MAGIC models feature-based object-driven selection, but does not combine this with selection of a spatial region of interest. Hence, it is not possible to focus on a particular location in the image. A closely related approach to our work is proposed by Vecera and O'Reilly [9]. Their model is based on the principles of parallel distributed processing (PDP) models of information processing. In their work they demonstrated the figure-ground segmentation as an interactive process.

\section{Approach}

The approach used in this work is inspired by, generally, the information processing in the human brain and more specifically along the two visual processing pathways, i.e., the ventral pathway and the dorsal pathway, of the visual system. Previous work has shown that attention emerges as a natural consequence of interaction between the two processing pathways and selects a specific location or object for further processing along the ventral channel [17][18]. In this work it is hypothesized that dorsal pathway learns the shape information of the patterns, in addition to encoding the spatial information. Here, the strategy is to segment the bottom-up saliency map and develop a segmented saliency map, under the guidance of top-down shape information, as an interactive process. The segmented saliency map should represent the spatial as well as identity information of one of the overlapped patterns. This information encapsulated in the segmented map can be used to modulate processing along the ventral channel in favor of that pattern.

The main thrust of this approach is on incremental development of a segmented saliency map that is based on an interactive process, 'recognition followed by segmentation, segmentation followed by recognition'. In this approach a patch of the bottom-up saliency map of the occluded patterns is selected and identified as a part of a specific pattern by matching with the stored templates. The identified pattern class is activated at the higher level. This higher level perception then guide the growth of 
segmented saliency maps along the spatial locations or contours that belong to the same pattern. This interactive process of incremental growth of segmented saliency map proceeds in small steps and lead to development of map that encode the spatial location as well as shape information of one of the occluded pattern. During testing when an overlapped pattern is presented to the model, the dorsal pathway develops the segmented saliency maps of the overlapped patterns. These maps are used to modulate the ventral pathway in favor of one of the overlapped patterns at a time and to recognize those patterns one by one.

This approach requires information to flow not only in bottom-up or top-down direction but all possible direction. In this work this kind of omnidirectional processing is realized by developing an interactive (recurrent, bidirectional) neural network. To keep the activation in control and to develop sparse representations in the model an inhibitory mechanism of $\mathrm{k}$-winners-take-all is employed.

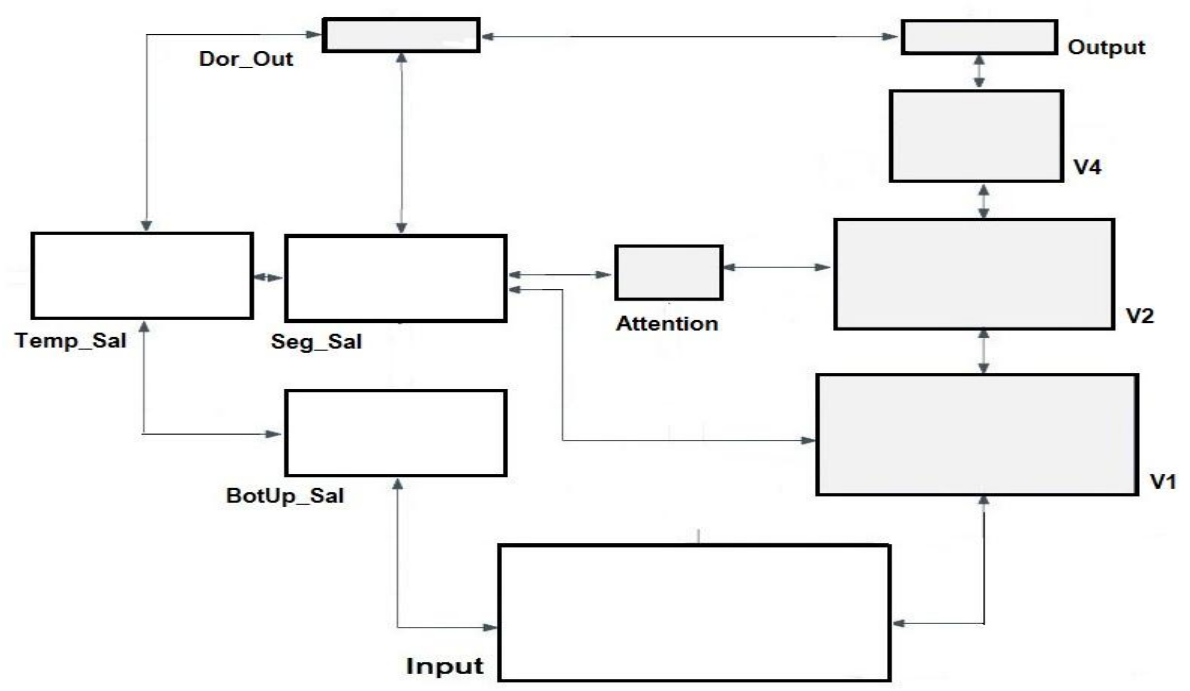

Fig. 2. The proposed biologically-inspired model

\section{The Model}

It is a hierarchical multi-layered neural network as illustrated in the figure 2. The model has two parallel processing channels which interact with each other through lateral connections among layers at appropriate level, and thus processing in one channel influences the processing in other. The two processing channels are named as ventral channel and dorsal channel after the names of visual information processing pathways in the human brain.

The Ventral Channel: This channel learns invariant representations of the input patterns while processing information along its hierarchical structure. The first layer in this pathway is named as V1. It is designed to detect oriented edge like 
biologically- inspired features from the input image. The units of this layer are arranged into groups such that each group processes a specific part of the input image. All the units within a group of V1 layer detect similar oriented edge like feature from the same spatial location, but for different orientation.

The next layer along the hierarchy is V2, which have larger receptive field than that of V1. The units of this layer extract complex features from the previous layer. As units of this layer, like V2 layer, are divided into groups therefore, somewhat week topographic representation of the input is still present at this stage.

The next layer is V4, which has the largest receptive field in the hierarchy that encompasses the whole input to the network. Each unit of this layer gets input from all units in the previous layer. Since it has a single receptive filed therefore this level does not possess any topographic information.

The Output layer is the last and highest processing level of this channel. It learns to assign the different representations of the same class at V4 to a single distinct output unit that represents this class.

The Dorsal Channel: The main functionality of this channel is to make ventral channel process one pattern at a time, in case more than one patterns are present in input. This channel encodes spatial information of the input patterns. It also learns the shape of the input pattern and used this information to guide segmentation of the overlapped patterns. This pathway interacts with the ventral pathway, at appropriate levels of processing stages, to modulate it in favor of specific locations and features. Since this pathway encode the spatial representation of the patterns, which is topographic in nature, therefore, in order to interact correctly with the ventral channel it must interact with those stages of the ventral pathway which keep, in addition to pattern specific information, topographic information of the input.

The first stage of processing in this channel is BotUp_Sal layer, which draw its input directly from the Input layer and develops bottom-up saliency map of the input. It is smaller in size than the Input layer and each unit of this layer receive input from a group of units from the Input layer. This connectivity pattern helps reduce (to some extent) shape distortion as well as position and size variations among patterns of the same class. This stage processes information on the basis of its connectivity patterns and does not perform any learning.

The next stage of this channel, Temp_Sal layer, is of the same size as BotUp_Sal and connected through it with a one to one connectivity pattern. Moreover, each unit of this layer is connected to eight close neighbors through self-connection. This layer allow only a very small number of units to be active at a given time, furthermore, the self-connectivity force the active units to appear in the form of one or two groups. Additionally, a mechanism of 'accommodation' is employed on the units of this specific layer. This mechanism causes a unit that is active for a while to get tired and die down for a short period of time, thereby giving way to other competing units to become active. In this way active group(s) of units seems to be moving along the contours of the pattern in the previous layer.

The next stage is Seg_Sal, which has the same size as the last two layers and connected to the Temp_Sal layer with one to one connectivity pattern between units. This layer has the large $\mathrm{k}$ value for $\mathrm{kWTA}$ mechanism comparing to the previous 
layer. In this layer an additional mechanism of 'hysteresis' is used, which causes active units to remain active for a short period of time even after removal of their excitatory input. In this way, this layer keeps the trace of moving activating patterns. The Attention layer is connected to the Seg_Sal and V2 layer and extracts its input from the both.

The Dor_Out layer is the highest processing layer of the dorsal channel. It associates template like spatial as well as shape representations of the input patterns that develop at the Temp_Sal and Seg_Sal layer, to a specific class. This layer receives bottom-up cues and in response generates top-down shape specific cues to guide the segmentation processes.

Interaction between the dorsal and ventral channels: The dorsal and ventral channels are connected to each other at three levels of pressings. The layers Seg_Sal, Attention and Dor_Out of the dorsal channel are connected to three layers V1, V2 and Output of the ventral channel. The Seg_Sal and Attention layer modulate the ventral channel for specific spatial locations, while the Dor_Out modulate the ventral channel for a specific class of patterns or features belongs to a specific class of patterns.

\subsection{Network Algorithm}

An interactive (bidirectional, recurrent) neural network is developed to realize the model. The network was developed in Emergent [19], using the biological plausible algorithm Leabra [20]. Each unit of the network had a sigmoid-like activation function. Learning in the network was based on a combination of Conditional Principal Component Analysis (CPCA), which is a Hebbian learning algorithm and Contrastive Hebbian learning (CHL), which is a biologically-based alternative to back propagation of error, applicable to bidirectional networks [20].

$$
\text { CPCA: } \Delta_{\text {hebb. }}=\varepsilon y_{j}\left(x_{i}-w_{i j}\right)
$$

$\varepsilon=$ learning rate

$x_{i}=$ activation of sending unit $i$

$y_{j}=$ activation of receiving unit $j$

$w_{i j}=$ weight from unit $i$ to unit $j \in[0,1]$

$$
\text { CHL: } \quad \Delta_{\text {err }}=\varepsilon\left(\mathrm{x}_{\mathrm{i}}^{+} \mathrm{y}_{\mathrm{j}}^{+}-\mathrm{x}_{\mathrm{i}}^{-} \mathrm{y}_{\mathrm{j}}^{-}\right)
$$

$x^{-}, y^{-}=$act when only input is clamped

$x^{+}, y^{+}=$act when also output is clamped

$$
\text { L_mix: } \Delta w_{i j}=\varepsilon\left[c_{\text {hebb }} \Delta_{h e b b}+\left(1-c_{\text {hebb }}\right) \Delta_{e r r}\right]
$$


$\mathrm{c}_{\mathrm{hebb}}=$ proportion of Hebbian learning

In Eq 3, L_mix represent the weight update as a result of learning that is a combination of Hebbian and error-driven learning. The $c_{\text {hebb }}$ value in L_mix based on the previous work [17].
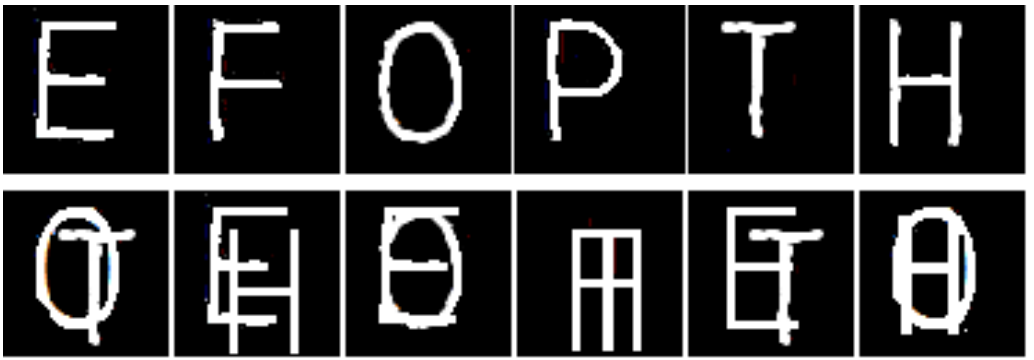

Fig. 3. Top row: Six English alphabet pattern classes used for simulations. Bottom row: Some of the test patters developed by overlapping the patterns

\section{$4 \quad$ Data for training and testing}

To simplify training and analysis of the results gray level images of six English alphabet patterns (fig. 3, top row) are used for simulations. Both machine printed as well as handwritten patterns are used as a data set for training. Each of the patterns is shifted in two steps in all eight directions, where length of the each step is four pixels. For testing, stimuli containing overlapped patterns were developed (fig. 3, bottom row). Such that, each stimulus contains two randomly selected patterns.

\section{$5 \quad$ Simulation and Results}

Training of the two network channels, i.e., the ventral and the dorsal channels was performed separately. The ventral channel learns the invariant representations of the eight input pattern classes on the basis of edge features extracted at the lowest level, i.e., V1 layer. The dorsal channel, on the other hand, learns templates representations of the eight input pattern classes. Each unit of Output and Dor_Out layer represent one pattern class in the order they appear in the top row of fig. 3. After completion of the training, the trained network is simulated on overlapped patterns. In the following section a few simulation results with brief analysis are presented to demonstrate the workability of the approach. The first two simulations show the interactive segmentation of the bottom-up saliency map for one of the overlapped pattern in the dorsal channel. The third simulation demonstrates the segmentation of saliency map for two overlapping patterns in a sequential manner, one after another. The fourth simulation result demonstrates the segmentation of the bottom-up saliency map at the 
dorsal channel as well as their corresponding modulation and recognition of individual patterns in the ventral channel, in a sequential manner.

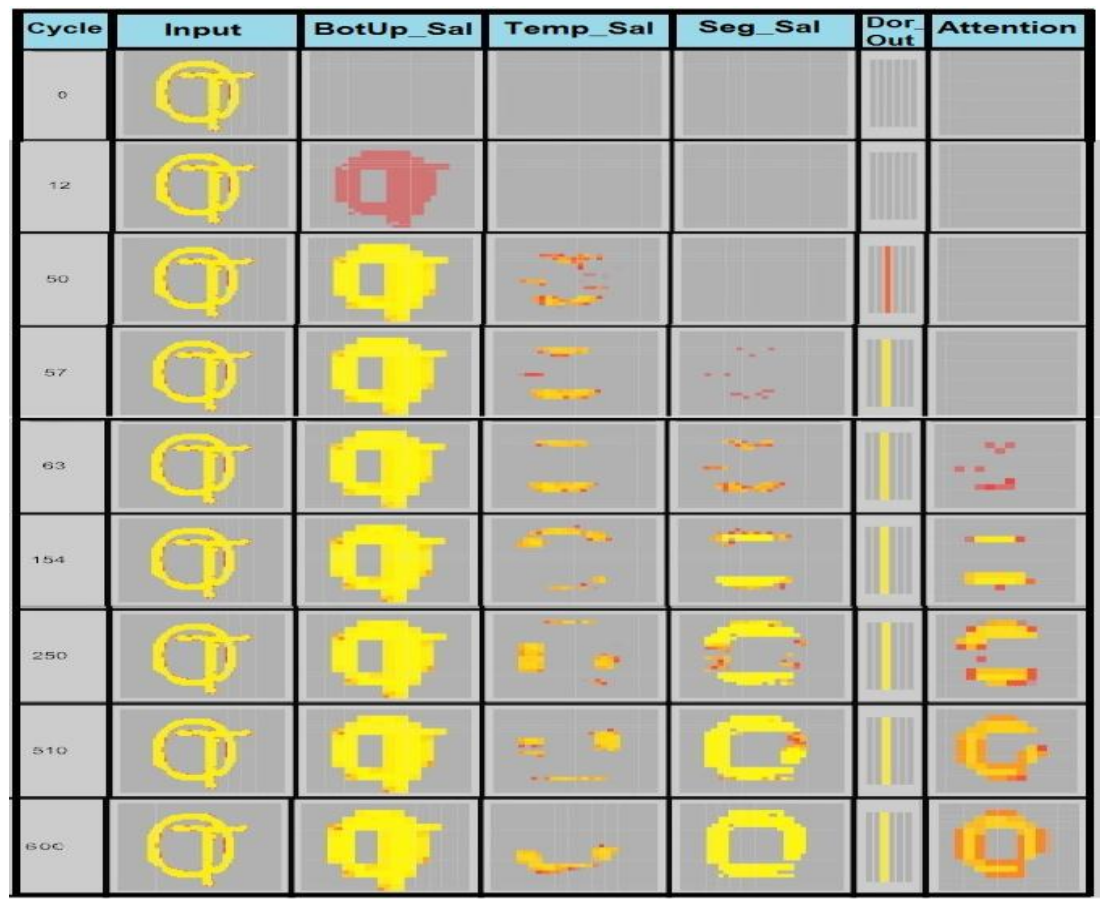

Fig. 4. Snapshots of activations in the various network layers (each layer is made up of a matrix of units, and the activation values of these matrices are shown here). The recorded changes in activation for different processing cycles illustrate how the top-down shape knowledge guide the bottom-up image-based information for interactive segmentation the saliency map in the dorsal channel. The segmented map represent one of the overlapping pattern at a time. For each graph, in the order from left to right, the columns represent: Number of processing cycle (how far computation of activation has gone), activations in Input layer, BotUp_Sal layer, Temp_Sal layer, Seg_Sal layer, Dor_Out layer and Attention layer of the network. Yellow (light) colors denote high activation values, red (dark) colors low activation. Gray (neutral) color means no activation

\subsection{Segmentation of bottom-up saliency map for one of the overlapped pattern in the dorsal channel}

Figure 4 shows the behavior of the dorsal channel when a stimulus is presented to the network. This stimulus contains two overlapped patterns ' $O$ ' and ' $T$ '. When is presented at the input layer, botom-up saliency map of the overlapped pattern is generated at the BotUp_Sal layer (cycle: 12). Next, Temp_Sal layer get activation from the BotUp_Sal layer, and due to low value of K for the KWTA relatively small number of its units become active. Due to self-connection, the active units in this layer appear in the form of one or two groups (cycle: 57-63). The Temp_Sal layer, on 
the basis of the similarity of the active groups with the learned pattern templates, activates the Dor_Out layer that represents, in this instance, the pattern ' $O$ '. The Dor_Out layer in return feedback to the Temp_Sal and Seg_Sal layers, and thus modulate these in favor of pattern ' $\mathrm{O}$ '. This interactive process proceeds in small steps and consequently active group of units at Temp_Sal layer moves over the spatial area representing pattern (obtaining input from saliency map of the ' $\mathrm{O}$ ' in the BotUp Sal layer) 'O' (57-600). In the meantime, the Seg_Sal layer keeps the traces of the moving groups at the Temp_Sal layer (cycle: 67-600). The Temp_Sal layer also interact with the Dor_Out layer and strongly influence it, therefore plays an important role in influencing the top-down signals. The resulting map at the Seg_Sal layer appears in the form of a rough template representing the pattern ' $O$ '. This map contains the spatial as well as shape information of the character ' $\mathrm{O}$ ' in the overlapped pattern, which help modulate the ventral channel processing in favor of this pattern. Attention layer, which serve as a communication bridge between two pathways for spatial modulation, also display the activation patterns of a specific shape.

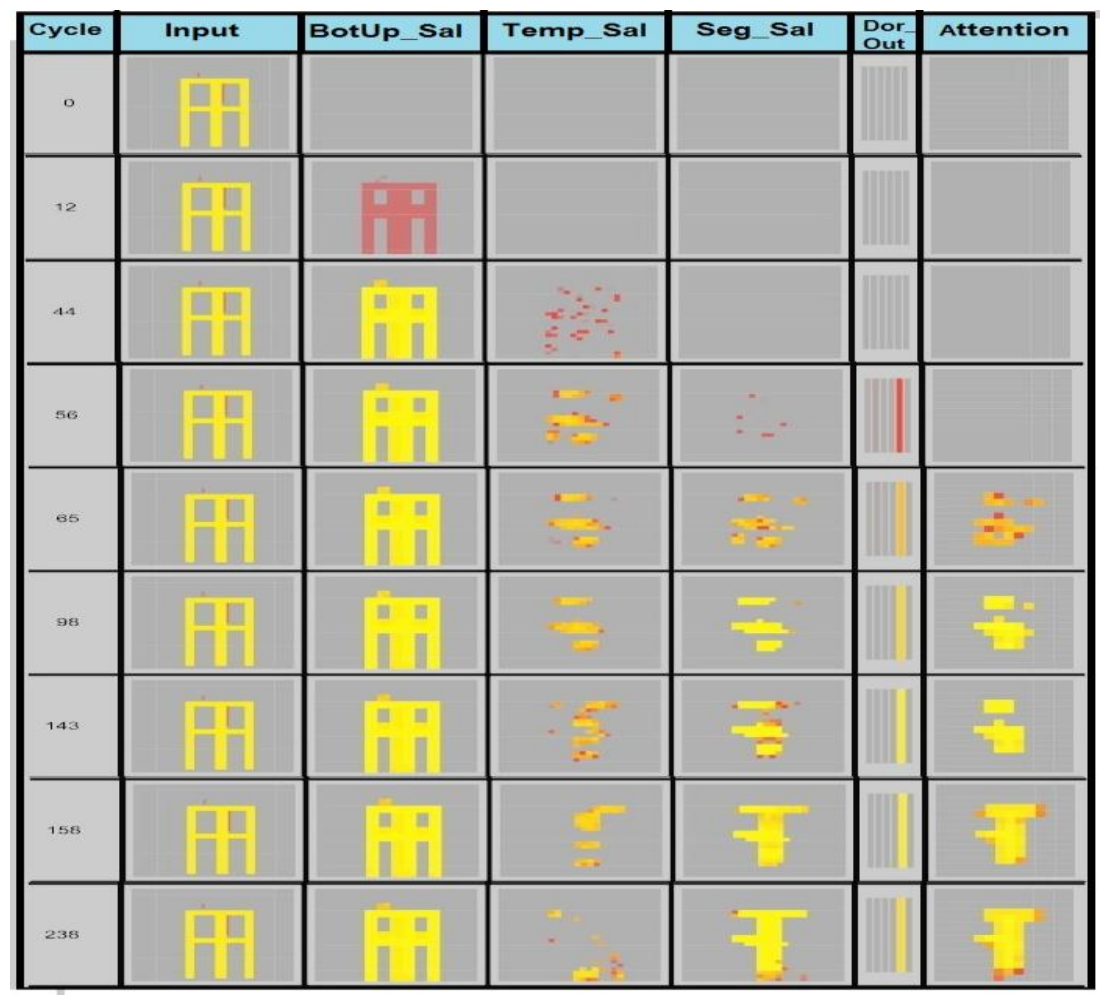

Fig. 5. Cycle-wise activation of various network layers while developing saliency map for one of the patterns through interactive processing: a complex pattern 


\subsection{Segmenting one of the bottom-up saliency map for one of the overlapped pattern: A complex overlapped pattern}

In this simulation, fig. 5, a stimulus that contains somewhat complex and ambiguous overlapped patterns is presented to the network. It is composed of two overlapping patterns ' $\mathrm{T}$ ' and ' $\mathrm{H}$ ', but their overlap created contours for characters ' $\mathrm{F}$ ' and ' $\mathrm{P}$ ' as

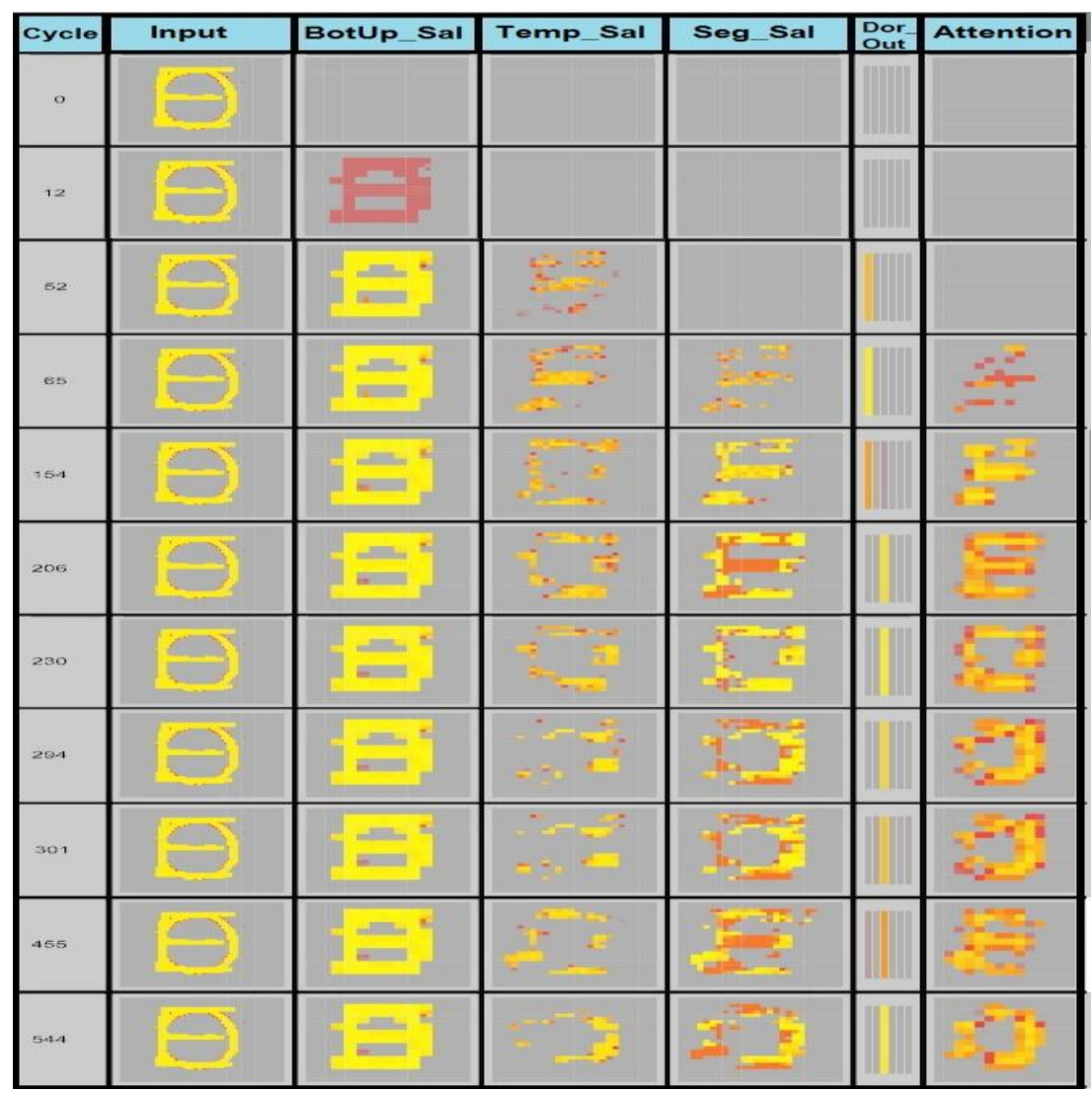

Fig. 6. Cycle-wise activation of various network layers while segmenting bottom-up saliency maps for overlapped patterns in a sequential manner, one by one.

well. Therefore, this stimulus can be segmented as one of the four possible classes of patterns.

When this stimulus is presented to the model, the BotUp_Sal layer activates the saliency map of the stimulus (cycle: 12-544) and gradually at the Temp_Sal layer a couple of groups of units become active (cycle: 44). The very first active groups of units at the Temp_Sal layer, in this case, matches the pattern ' $T$ ' and as a consequence, activates the corresponding representative unit of this class at the Dor_Out layer. The Seg_Sal layer, like in the previous case, keeps traces of activations of the Temp_Sal layer. The interaction among these three layers leads to 
and segmentation of bottom-up saliency map incremental development of a saliency map in favor of pattern ' $T$ '. The segmentation results are continuously feed to the Attention layer (cycle: 65-236).

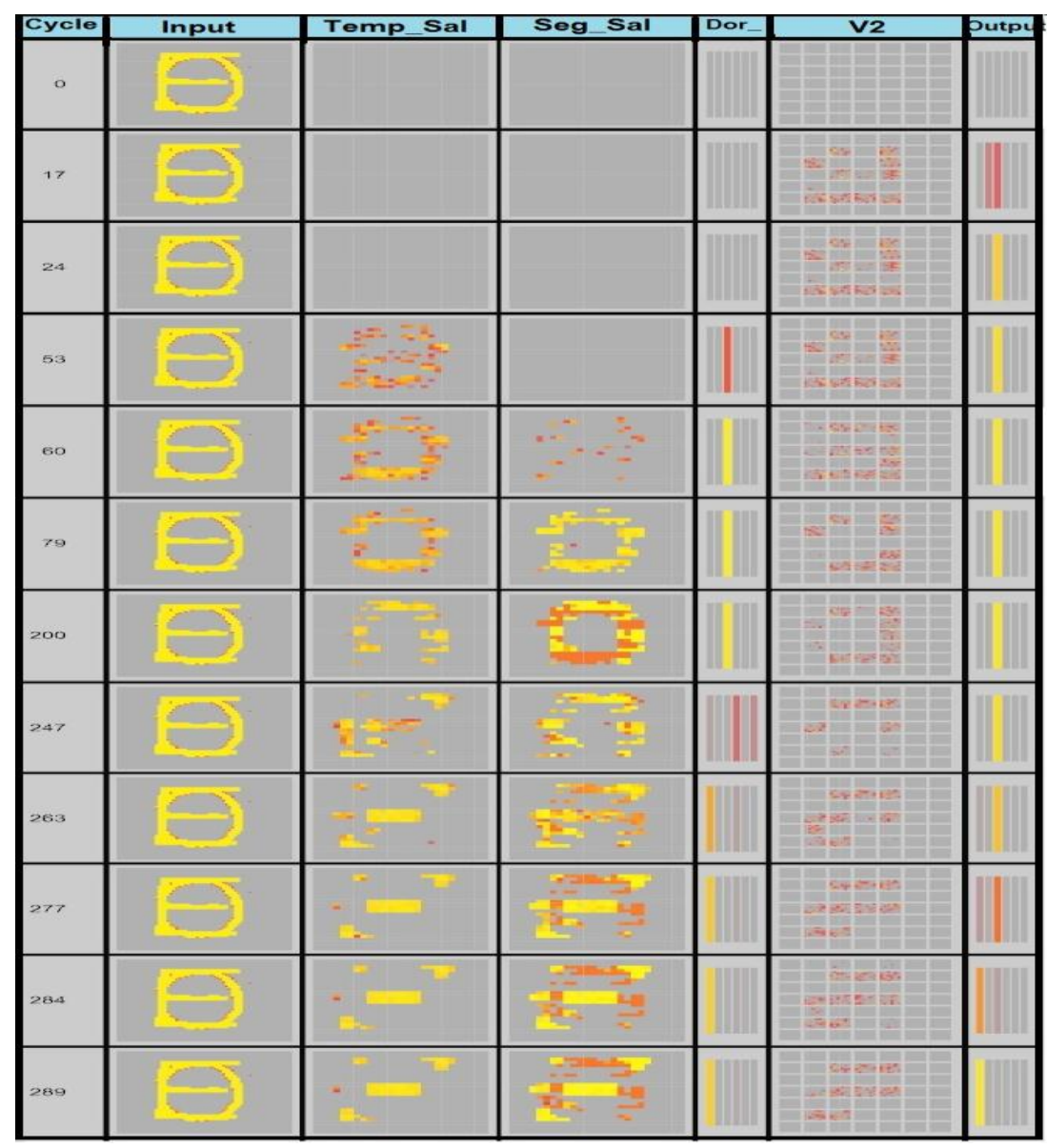

Fig. 7. Cycle-wise activation of various network layers while segmenting bottom-up saliency map for overlapped patterns in a sequential manner, one by one, and corresponding modulation and recognition of individual patterns in the ventral channel

\subsection{Segmenting both of the overlapped patterns in saliency map}

Figure 6 shows the simulation results when the dorsal channel generates the saliency maps for two overlapped patterns one by one. The stimulus used is composed of two overlapped patterns ' $\mathrm{E}$ ' and ' $\mathrm{O}$ '. When it is presented to the model, the BU_Map 
layer generates a bottom-up saliency map (cycle: 12-544). The Temp_Sal layer activates a chunk of this saliency map which subsequently identified as the pattern ' $E$ ' at the Dor_Out layer (cycle: 52-154). As a result, the top-down signals are generated that modulate the Temp_Sal and Seg_Sal layers in favor of pattern 'E'. As bottom-up signals contain the activation patterns of 'E', therefore, Seg_Sal grows and develops a map for ' $E$ ' due to interaction between top-down and bottom-up influences (cycle: 65-206). The attention layer, which serves as a communicating layer between two channels, also shows the corresponding saliency maps (cycle: 65-206). Meanwhile, some of the active units at the Seg_Sal layer start getting tired and thus give way to other competing units to become active (cycle: 154-206). Most probably the newly activated units belong to the other pattern ' $O$ ', because units that represents ' $E$ ' were already active. At this moment the newly active units support the pattern ' $\mathrm{O}$ ' and thus forced the Dor_Out layer to generate top-down signals that favor this new pattern (cycle: 154). Consequently, the top-down and bottom-up interaction leads to segmentation of bottom-up saliency map and incremental development of a segmented map that represents the pattern 'O' (cycle: 230-544). This process goes on, such that interactive processing and tiring mechanism of units lead to segmentation of bottom-up saliency map and incremental development of saliency maps for each pattern one after another.

\subsection{Segmentation in dorsal and recognition in the ventral channel}

In this simulation segmentation of the bottom-up saliency map by interactive processing in the dorsal channel and, consequently, sequential recognition of overlapped patterns in the ventral channel is demonstrated. The stimulus used is composed of two overlapped patterns, i.e., pattern ' $E$ ' and ' $O$ '. On presentation of the stimulus, the BU_Sal layer generates the bottom-up saliency map for the overlapped patterns and the Seg_Sal layer develops the segmented saliency maps of the occluded patterns one by one, by using tiring function of the units, as in the previous simulation. The two channels interact with each other at three levels and consequently the ventral channel recognizes the patterns one by one in a sequential manner.

Consider the fig 7, first the Seg_Sal develops the saliency map for pattern 'O' (cycle: 60-200). The dorsal channel modulates the processing of the ventral channel at three levels. The Interact_map and the Attention modulate the activation of V1 and V2 layers for specific spatial locations and, Dor_Out layer interact modulates the Output layer of the ventral channel which in turn provides the feature based modulation of the ventral channel for the pattern ' $O$ '. Consequently, the ventral channel identify one of the overlapped pattern as ' $\mathrm{O}$ '. Next, when the saliency map for the ' $E$ ' is developed and modulation of the ventral channel is performed the Output layer starts to identify the second pattern as ' $E$ '. 


\section{Conclusion}

In this paper a biologically inspired model for the recognition of overlapped patterns is presented. It is hypothesized that, in addition to encoding the spatial information, the dorsal channel learns the shape information of the patterns. An interactive process, based on the bottom-up information and top-down shape information, segment the bottom-up saliency map and develop saliency maps for each of the overlapped patterns. The segmented maps contain the spatial as well as shape information of the respective patterns in the input. The interaction between the dorsal and the ventral pathway modulate the processing in favor of that pattern.

The computers simulations with the model demonstrate that it provides a satisfactory solution to the problem of overlapped patterns recognition, which have little bottom-cues for segmentation. The model produces a behavior that is in accordance to the known human behavior for recognition of overlapped patterns.

\section{References}

[1] I. Biederman, "Recognition-by-components: A theory of human image understanding.," Psychological review, vol. 94, no. 2, p. 115, 1987.

[2] D. Marr and W. Vision, "Freeman and Company," San Francisco, 1982.

[3] S. Palmer and I. Rock, "Rethinking perceptual organization: The role of uniform connectedness," Psychonomic Bulletin \& Review, vol. 1, no. 1, pp. 29-55, 1994.

[4] S. Ullman, "Aligning pictorial descriptions: An approach to object recognition* 1," Cognition, vol. 32, no. 3, pp. 193-254, 1989.

[5] D. G. Lowe, "Perceptual Organization and Visual Recognition.," DTIC Document, 1984.

[6] A. Needham, "Object Recognition and Object Segregation in 4.5-Month-Old Infants* 1," Journal of experimental child psychology, vol. 78, no. 1, pp. 3-22, 2001.

[7] M. A. Peterson, "Object recognition processes can and do operate before figure-ground organization," Current Directions in Psychological Science, vol. 3, no. 4, pp. 105-111, 1994.

[8] S. P. Vecera and M. J. Farah, "Is visual image segmentation a bottom-up or an interactive process?," Attention, Perception, \& Psychophysics, vol. 59, no. 8, pp. 1280-1296, 1997.

[9] S. P. Vecera and R. C. O'reilly, "Figure-ground organization and object recognition processes: An interactive account.," Journal of Experimental Psychology: Human Perception and Performance, vol. 24, no. 2, p. 441, 1998

[10] L. G. Ungerleider and J. V. Haxby, "What and where in the human brain.," Current opinion in neurobiology, vol. 4, no. 2, p. 157, 1994.

[11] A. Sereno and J. Maunsell, "Shape selectivity in primate lateral intraparietal cortex,” J. Exp. Psychol. Hum. Percept. Perform, vol. 12, pp. 388-391, 1987. 
[12] S. R. Lehky and A. B. Sereno, "Comparison of shape encoding in primate dorsal and ventral visual pathways," Journal of neurophysiology, vol. 97, no. 1, p. 307, 2007.

[13] M. C. Mozer, R. S. Zemel, M. Behrmann, and C. K. I. Williams, "Learning to segment images using dynamic feature binding," Neural Computation, vol. 4, no. 5, pp. 650-665, 1992.

[14] M. Behrmann, "Discovering and using perceptual grouping principles in visual information processing," in Proceedings of the Fourteenth Annual Conference of the Cognitive Science Society, 1992, vol. 14, p. 283.

[15] S. Grossberg, "A Solution of the Figure-Ground Problem for Biological Vision," Neural Networks, vol. 6, pp. 463-483, 1993.

[16] M. Behrmann, R. S. Zemel, and M. C. Mozer, "Object-based attention and occlusion: Evidence from normal participants and a computational model.," Journal of Experimental Psychology: Human Perception and Performance, vol. 24, no. 4, p. 1011, 1998.

[17] M. Saifullah, "Exploring Biologically-Inspired Interactive Networks for Object Recognition," 2011.

[18] M. Saifullah and R. Kovordányi, "Emergence of attention focus in a biologically-based bidirectionally-connected hierarchical network," Adaptive and Natural Computing Algorithms, pp. 200-209, 2011.

[19] B. Aisa, B. Mingus, and R. O'Reilly, "The emergent neural modeling system," Neural networks, vol. 21, no. 8, pp. 1146-1152, 2008.

[20] R. C. O'Reilly and Y. Munakata, Computational explorations in cognitive neuroscience: Understanding the mind by simulating the brain. The MIT Press, 2000 . 